\title{
Numerical Simulation Dynamics Research on Selective Reduction in Reaction Vessel of Fine Iron and Carbon Mixture
}

\author{
Lan Xiaowen \\ School of Information Engineering, Inner Mongolia University of Science \\ \&Technology, Baotou, China \\ lanxiaowenning@163.com
}

\begin{abstract}
With the development of computer technology, computer numerical simulation has become one of the three major scientific methods in metallurgy field. Its basic idea) is to achieve the simulation of metallurgical process and the design optimization through mathematical modeling, numerical dissertation and computer programming Referring to the reaction model of theory of porous media within gas and solid, this research focuses on the selective reduction process in mixture vessel of fine ironand carbon, taking into consideration of heat transfer equation of porous medium, the ate equation of carbon gasified reaction, the equation of continuity, and the momentum equation and so on, and establishes a micro dynamics mathematical model of the Nelective reduction process of fine iron and carbon mixture under the non-uniform ternperature and hon-equal-pressure conditions. Based on this model, the analysis of micro-dymamics of selective reduction process can be conducted; it can be applied to analyses of the effects of mass Transfer in gas phase, solid-state diffusion and surface chemical reaction to the selective reduction process, as well as the interaction between two different oxides and complex compounds; the enhance means of speed control can also be obtained.
\end{abstract}

Keywords: Numerical Simulation, Selective Reduction, Dynamics, Reaction Vessel

\section{Introduction}

Selective reduction is current research focus in the field of metallurgy. There are rich references from thermodynamics to the dynamics, from micro to macro dynamics, from experiment to mathematical models, from process research to microstructure observations, among which micro-dynamics studies have taken into account the factors such as porous media, heat transfer, quality, and interface and so on, and d some systemic researches have been conducted in dynamics experiments and mathematical models.

The computer numerical simulation research on selective reduction can have easy access to the relatively mature experimental data to change the related process parameters. Meanwhile, based on some original data which are closer to the primary state, the research makes use of mathematical model to calculate the variables related, such as heat transfer, mass transfer, and momentum transfer. In this way, the quantitative analysis of related variables can be finished before the start of the actual selective reduction process. Therefore, it is convenient to adjust the craft plan, optimize parameter, and guarantee the success rate. As a result, it is of vital importance in shortening the production cycle, reducing costs and improving economic efficiency. 


\section{The Experimental Device for Selective Reduction of Fine Iron and Carbon Mixture in Reaction Vessel}

The selective reduction device as shown in Figure 1:

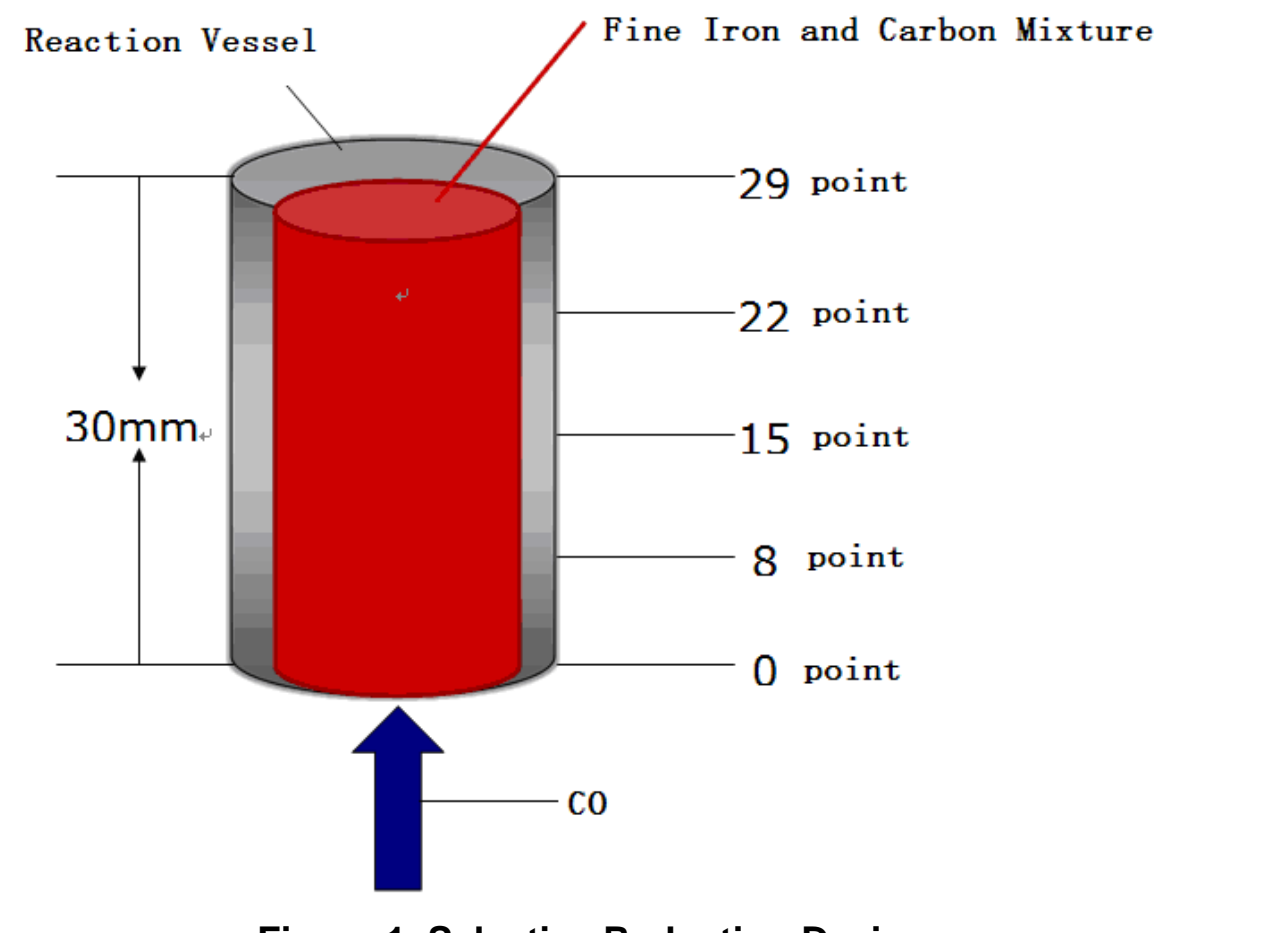

Figure 1. Selective Reduction Device

The selective-reduction device is a reaction vessel containing iron ore with the height $30 \mathrm{~mm}$, which is evenly divided into 5 points. $\mathrm{CO}$ is input from the bottom of the reaction vessel, with heating temperature at $100{ }^{\circ} \mathrm{c}$ at the bottom. $\mathrm{CO}_{2}$ is output from the top of the vessel, with the temperature at the top of and the component of carbon monoxide and carbon dioxide having no boundary effect. The chemical reaction equation is:

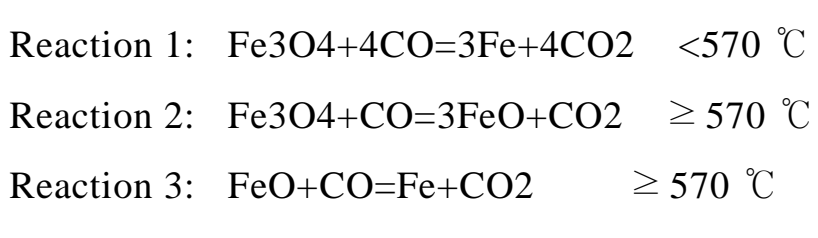

Three reactions are carried out in the order of reaction 1 , reaction 2 , and then the reaction

\section{Mathematical Model}

(1) Internal heat transmission equation porous medium

$$
\frac{\partial}{\partial t}\left(\rho_{m} C_{p m} T\right)+\frac{1}{r} \frac{\partial}{\partial r}\left(r\left(\rho_{g} V_{0} C_{p g} T-K_{e f f m} \frac{\partial T}{\partial r}\right)\right)=-\sum_{i} \dot{R}_{i} \Delta H_{i}
$$

(2) Dynamics equation of chemical reaction out of phase 
Reduction of iron-oxide compounds

With $\mathrm{CO}$ as the reducing agent, reducing reaction is as follows:

$$
\begin{array}{lr}
3 \mathrm{Fe}_{2} \mathrm{O}_{3}+\mathrm{CO}=2 \mathrm{Fe}_{3} \mathrm{O}_{4}+\mathrm{CO}_{2} \\
\mathrm{Fe}_{3} \mathrm{O}_{4}+4 \mathrm{CO}=3 \mathrm{Fe}+4 \mathrm{CO}_{2} & <570{ }^{\circ} \mathrm{C} \\
\mathrm{Fe}_{3} \mathrm{O}_{4}+\mathrm{CO}=3 \mathrm{FeO}+\mathrm{CO}_{2} & \geq 570{ }^{\circ} \mathrm{C}
\end{array}
$$$$
\mathrm{FeO}+\mathrm{CO}=\mathrm{Fe}+\mathrm{CO}_{2} \quad \geq 570{ }^{\circ} \mathrm{C}
$$

With $\mathrm{CO}$ as the reducing agent, the equation of the reaction rate is as follows:

$$
\begin{gathered}
\dot{R}_{i}^{C O}=S_{f i} n_{i} 4 \pi \bar{r}_{i}^{2} k_{i}^{C O}\left(C_{C O}-\frac{C^{C O}}{k_{C i}^{C O}}\right) \\
k_{i}^{C O}=k_{i 0}^{C O} \exp \left(\frac{-\Delta E_{j C O}}{R T}\right)
\end{gathered}
$$

With $\mathrm{H}_{2}$ as the reducing agent, the reducing reactions and rate equation are similar to the above.

Total reaction rate of iron oxifle edúction iscalculated as follows:

$$
\dot{R}_{i}=\dot{R}_{C}^{C O}+\dot{R}_{i}^{H_{2}}
$$

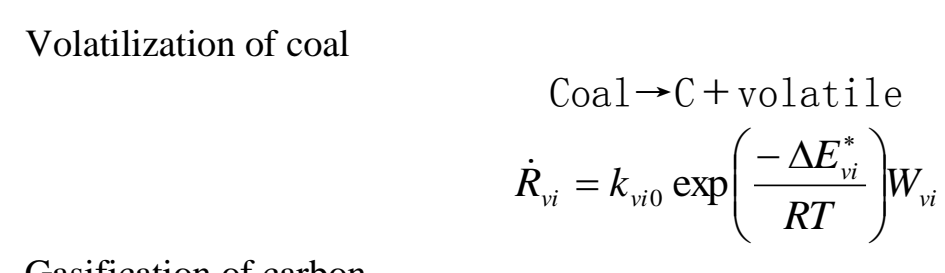

\section{Gasification of carbon}

The gasification reaction is as follows :

$$
\mathrm{C}+\mathrm{CO}_{2}=2 \mathrm{CO}
$$

The readtion rate equation of carbon gasification:

$$
\begin{array}{r}
\dot{R}_{c}^{\mathrm{CO}_{2}}=S_{c} n_{c} 4 \pi \bar{r}_{c}^{2} k_{c}^{C O_{2}}\left(C_{\mathrm{CO}_{2}}-\frac{C_{C O}^{2}}{K_{E c}^{C O_{2}}}\right) \\
k_{c}^{C O_{2}}=k_{c 0}^{C O_{2}} \exp \left(\frac{-\Delta E_{c O_{2}}^{*}}{R T}\right)
\end{array}
$$

Water gas reaction is as follows:

$$
\mathrm{C}+\mathrm{H}_{2} \mathrm{O}=\mathrm{CO}+\mathrm{H}_{2}
$$


Water gas reaction rate equation:

$$
\dot{R}_{c}^{\mathrm{H}_{2} \mathrm{O}}=S_{c} n_{c} 4 \pi \bar{r}_{c}^{2} k_{c}^{\mathrm{H}_{2} \mathrm{O}}\left(C_{\mathrm{H}_{2} \mathrm{O}}-\frac{C_{C O} \times C_{\mathrm{H}_{2}}}{K_{E c}^{\mathrm{H}_{2} \mathrm{O}}}\right)
$$

Total gasification reaction rates are calculated as follow:

$$
\dot{R}_{c}=\dot{R}_{c}^{\mathrm{CO}_{2}}+\dot{R}_{c}^{\mathrm{H}_{2} \mathrm{O}}
$$

Size changes of pulverized coal particles in the heating process are as follows:

$$
\bar{r}_{c}=\bar{r}_{c}^{0}\left(1+\gamma \times \exp \left(\frac{-(T-650)^{2}}{2 \times 300^{2}}\right)\right)
$$

$\gamma$ is constant, value is 0.18

(3) gaseous phase conservation equations

in the gaseous phase, these components are $\mathrm{CO} 、 \mathrm{CO}_{2} 、 \mathrm{H}_{2}, \mathrm{H}_{2} \mathrm{O} 、 \mathrm{CH}_{4} 、$ Tar etc, for each component continuity equations yere establishedas follows :

$$
\frac{\partial}{\partial t}\left(\phi \rho_{j}\right)+\frac{1}{r} \frac{\partial}{\partial r}\left(r\left(\hat{V}_{j} \rho_{j}-\frac{\phi}{\tau} \dot{D}_{\text {effj }} \frac{\partial \rho_{j}}{\partial r}\right)\right)=-\sum_{i} \dot{N}_{i j} M_{j}
$$

Formula for calculating the effective diffusion coefficient of $\mathrm{j}$ in the gas mixture components:

(4) Continuity equation

$$
D_{f f j}=\left(1-y_{j}\right)\left(\sum_{\substack{i=1 \\ i \neq j}} \frac{y_{i}}{D_{i j}}\right)^{-1}
$$

Gas-phase contnuity equation is as follows:

$$
\frac{\partial}{\partial t}\left(\phi \rho_{g}\right)+\frac{1}{r} \frac{\partial}{\partial r}\left(r V_{0} \rho_{g}\right)=-\sum_{j} \sum_{i} \dot{N}_{i j} M_{j}
$$

(5) Momentum equation

Vapor-momentum equation is as follows

$$
\frac{\partial}{\partial t}\left(\rho_{g} \frac{V_{0}}{\phi}\right)=-\frac{\partial P}{\partial r}-\alpha \mu V_{0}
$$

Where in :

$$
\alpha=150 \frac{(1-\phi)^{2}}{\phi^{3}\left(S_{f a} d_{p}\right)^{2}}
$$




$$
\mu=\frac{\sum_{j} M_{j}^{1 / 2} y_{j} \mu_{j}}{\sum_{j} M_{j}^{1 / 2} y_{j}}
$$

(6) The ideal gas equation

In the context of the given temperature and pressure, gas can be considered to be incompressible and meets the ideal gas equation of State

$$
P=\frac{\rho_{g}}{\bar{M}_{g}} R T
$$

\section{Discrete Solutions}

(1) Discretization of heat transmission equation in porous medium

$$
\frac{\partial}{\partial t}\left(\rho_{m} C_{p m} T\right)+\frac{1}{r} \frac{\partial}{\partial r}\left(r\left(\bar{\rho}_{g} V_{0} C_{p g} T-K_{e f f m} \frac{\partial T}{\partial r}\right)\right)=-\sum_{i} \dot{R}_{i} \Delta H_{i}
$$

With $S_{T}$ as the source item, $S_{T}=-\sum \dot{R}_{i} \Delta H_{i}$

$$
\frac{\partial}{\partial t}\left(\rho_{m} C_{p(r)} T\right)+\frac{1}{r} \frac{\partial}{\partial r}\left(r\left(\hat{\rho}_{g} C_{p g} V_{a} T-K_{e f f m} \frac{\partial T}{\partial r}\right)\right)=S_{T}
$$

For internal nodes $p$, Integration in the body under its control points

$$
\int \frac{\partial}{\partial t}\left(\rho_{m} C_{p m} T\right) d V_{+}+\frac{1}{r} \frac{\partial}{\partial r}\left(r\left(\rho_{g} C_{p g} V_{a} T-K_{e f f m} \frac{\partial T}{\partial r}\right)\right) d V=\int S_{T} d V
$$

Where in :

$$
\begin{gathered}
\int \frac{\partial}{\partial t}\left(\rho_{m} C_{p m} T\right) d V=\frac{\rho_{m}^{1} C_{p m}^{1} T_{P}^{1}-\rho_{m}^{0} C_{p m}^{0} T_{P}^{0}}{\Delta \tau} \Delta V \\
\int S_{T} d V=S_{T}^{0} \Delta V \\
\int \frac{1}{r} \frac{\partial}{\partial r}\left(r\left(\rho_{g} C_{p g} V_{a} T-K_{e f f m} \frac{\partial T}{\partial r}\right)\right) d V=J_{e}^{0} A_{e}-J_{w}^{0} A_{w} \\
J_{e}^{0}=\left(\rho_{g} C_{p g} V_{a} T-K_{e f f m} \frac{\partial T}{\partial r}\right)_{e}^{0}=\left(\rho_{g} C_{p g} V_{a}\right)_{e} T_{P}^{0}-\frac{\left(\rho_{g} C_{p g} V_{a}\right)_{e}}{\exp \left(P_{e}\right)-1}\left(T_{E}^{0}-T_{P}^{0}\right) \\
J_{w}^{0}=\left(\rho_{g} C_{p g} V_{a} T-K_{e f f m} \frac{\partial T}{\partial r}\right)_{w}^{0}=\left(\rho_{g} C_{p g} V_{a}\right)_{w} T_{P}^{0}-\frac{\left(\rho_{g} C_{p g} V_{a}\right)_{w} \exp \left(P_{w}\right)}{\exp \left(P_{w}\right)-1}\left(T_{P}^{0}-T_{W}^{0}\right) \\
\Delta V=r \Delta r \\
A_{e}=r_{e}, \quad A_{w}=r_{w}
\end{gathered}
$$


hypothesis :

$$
\begin{gathered}
F_{e}=\left(\rho_{g} C_{p g} V_{a}\right)_{e} A_{e}, \quad F_{w}=\left(\rho_{g} C_{p g} V_{a}\right)_{w} A_{w} \\
D_{e}=\frac{\left(K_{e f f m}\right)_{e}}{(\delta r)_{e}} A_{e}, \quad D_{w}=\frac{\left(K_{e f f m}\right)_{w}}{(\delta r)_{w}} A_{w} \\
P_{e}=\frac{F_{e}}{D_{e}}, \quad P_{w}=\frac{F_{w}}{D_{w}} \\
a_{E}=\frac{F_{e}}{\exp \left(P_{e}\right)-1}, \quad a_{W}=\frac{F_{w} \exp \left(P_{w}\right)}{\exp \left(P_{w}\right)-1}
\end{gathered}
$$

then :

$$
\frac{\rho_{m}^{1} C_{p m}^{1} \Delta V}{\Delta \tau} T_{P}^{1}=a_{E} T_{E}^{0}+a_{W} T_{W}^{0}+\left(F_{w}-F_{e}-a_{E} q_{W}+\frac{\rho_{m}^{0} C_{p m}^{0} \Delta V}{\Delta \tau}\right) T_{P}^{0}+S_{T}^{0} \Delta V
$$

(2) Discretization of gas-phase equations

$$
\frac{\partial}{\partial t}\left(\phi \bar{\rho}_{j}\right)+\frac{1}{r} \frac{\partial}{\partial r}\left(r\left(V_{0} \hat{\rho}_{j}-\frac{\phi}{\tau} D_{\text {eeff }} \frac{\partial \bar{\beta}_{j}}{\partial r}\right)\right)=-\sum_{i} \dot{N}_{i j} M_{j}
$$

Can be written as:

$$
\frac{\partial}{\partial t}\left(\rho_{g} Y_{j}\right)+\frac{1}{r} \frac{\partial}{\partial r}\left(r\left(\rho_{g} V_{a} Y_{j}-\Gamma_{j} \frac{\partial Y_{j}}{\partial r}\right)\right)=S_{j}
$$

For internal hodes $\mathrm{p}$, integration in the body under its control points

$$
\int \frac{\partial}{\partial t}\left(\rho^{Y}\right) d V+\int \frac{1}{r} \frac{\partial}{\partial r}\left(r\left(\rho_{g} V_{a} Y_{j}-\Gamma_{j} \frac{\partial Y_{j}}{\partial r}\right)\right) d V=\int S_{j} d V
$$

Where in

$$
\begin{gathered}
\int \frac{\partial}{\partial t}\left(\rho_{g} Y_{j}\right) d V=\frac{\rho_{g}^{1} Y_{j P}^{1}-\rho_{g}^{0} Y_{j P}^{0}}{\Delta \tau} \Delta V \\
\int S_{j} d V=S_{j}^{0} \Delta V \\
\int \frac{1}{r} \frac{\partial}{\partial r}\left(r\left(\rho_{g} V_{a} Y_{j}-\Gamma_{j} \frac{\partial Y_{j}}{\partial r}\right)\right) d V=J_{e}^{0} A_{e}-J_{w}^{0} A_{w}
\end{gathered}
$$

hypothesis :

$$
J_{e}^{0}=\left(\rho_{g} V_{a} Y_{j}-\Gamma_{j} \frac{\partial Y_{j}}{\partial r}\right)_{e}^{0}=\left(\rho_{g} V_{a}\right)_{e} Y_{j P}^{0}-\frac{\left(\rho_{g} V_{a}\right)_{e}}{\exp \left(P_{e}\right)-1}\left(Y_{j E}^{0}-Y_{j P}^{0}\right)
$$




$$
\begin{gathered}
J_{w}^{0}=\left(\rho_{g} V_{a} Y_{j}-\Gamma_{j} \frac{\partial Y_{j}}{\partial r}\right)_{w}^{0}=\left(\rho_{g} V_{a}\right)_{w} Y_{j P}^{0}-\frac{\left(\rho_{g} V_{a}\right)_{w} \exp \left(P_{w}\right)}{\exp \left(P_{w}\right)-1}\left(Y_{j p}^{0}-Y_{j w}^{0}\right) \\
\Delta V=r \Delta r, \quad A_{e}=r_{e}, \quad A_{w}=r_{w} \\
F_{e}=\left(\rho_{g} V_{a}\right)_{e} A_{e}, \quad F_{w}=\left(\rho_{g} V_{a}\right)_{w} A_{w} \\
D_{e}=\frac{\left(\Gamma_{j}\right)_{e}}{(\delta r)_{e}} A_{e}, \quad D_{w}=\frac{\left(\Gamma_{j}\right)_{w}}{(\delta r)_{w}} A_{w} \\
P_{e}=\frac{F_{e}}{D_{e}}, \quad P_{w}=\frac{F_{w}}{D_{w}} \\
a_{E}=\frac{F_{e}}{\exp \left(P_{e}\right)-1}, \quad a_{W}=\frac{F_{w} \exp \left(P_{w}\right)}{\exp \left(P_{w}\right)-1}
\end{gathered}
$$

then :

$$
\frac{\rho_{g}^{1} \Delta V}{\Delta \tau} Y_{j P}^{1}=a_{E} Y_{j E}^{0}+a_{W} Y_{j W}^{0}+\left(F_{w}-F_{e}-a_{E} \bullet a_{W} \frac{\rho_{g}^{0} \Delta V}{\Delta \tau}\right) Y_{j P}^{0}+S_{j}^{0} \Delta V
$$

(3) Discretization of continuity equations

$$
\frac{\partial}{\partial t}\left(\phi \bar{\rho}_{g}\right)+\frac{\partial}{r} \frac{\partial}{\partial r}\left(r V_{\sigma} \bar{\rho}_{g}\right)=-\sum_{j} \sum_{i} \dot{N}_{i j} M_{j}
$$

Can be written as:

hypothesis :

$$
\frac{\partial}{\partial t}(\rho g)+\frac{1}{r} \frac{\partial}{\partial r}\left(r \rho_{g} V_{a}\right)=S
$$

$S$ as the source item, $\$=-\sum_{j} \sum_{i} \dot{N}_{i j} M_{j}$, then :

Wherein:

$$
\begin{gathered}
\int \frac{\partial}{\partial t}\left(\rho_{g}\right) d V=\frac{\rho_{g}^{1}-\rho_{g}^{0}}{\Delta \tau} \Delta V \\
\int \frac{1}{r} \frac{\partial}{\partial r}\left(r \rho_{g} V_{a}\right) d V=\left(\rho_{g} V_{a}\right)_{e} A_{e}-\left(\rho_{g} V_{a}\right)_{w} A_{w}=F_{e}-F_{w} \\
\int S d V=S^{0} \Delta V
\end{gathered}
$$

then : 


$$
\frac{\rho_{g}^{1}-\rho_{g}^{0}}{\Delta \tau} \Delta V+F_{e}-F_{w}=S^{0} \Delta V
$$

(4) Discretization of momentum equation

$$
\frac{\partial}{\partial t}\left(\bar{\rho}_{g} \frac{V_{0}}{\phi}\right)=-\frac{\partial P}{\partial r}-\alpha \mu V_{0}
$$

make :

then :

$$
\alpha^{\prime}=150 \frac{(1-\phi)^{2}}{\phi^{2}\left(S_{f a} d_{p}\right)^{2}}
$$$$
\frac{\partial}{\partial t}\left(\bar{\rho}_{g} V_{a}\right)=\frac{\partial P}{\partial r}-\alpha^{\prime} \mu V_{a}
$$

For internal nodes $\mathrm{p}$, integration in the body under its control points

then :

$$
\int \frac{\partial}{\partial t}\left(\bar{\rho}_{g} V_{a}\right) d V=\int\left(\frac{\partial P}{\partial r}-\alpha^{\prime} \mu V_{a}\right) d V
$$

$$
\frac{\bar{\rho}_{g}^{\mathrm{lo}} \Delta V}{\Delta \tau} V_{a P}^{1}=P_{e} A_{e}-P_{w} A_{w}+\left(\frac{\bar{\rho}_{g}^{0}}{\Delta \tau} \Delta V-\alpha^{\prime} \mu \Delta V\right) V_{a P}^{0}
$$

The result can be obtained with discrete staggered grid solution.

\section{Calculation Resuls}

Based on the mathematical model above, the research developed a calculation program with Visual $\mathrm{C}++$ and a preliminary simulation was conducted in the fine iron reducing pocess under the one-way heating condition. The whole procedure is shown in Figure 2 . 


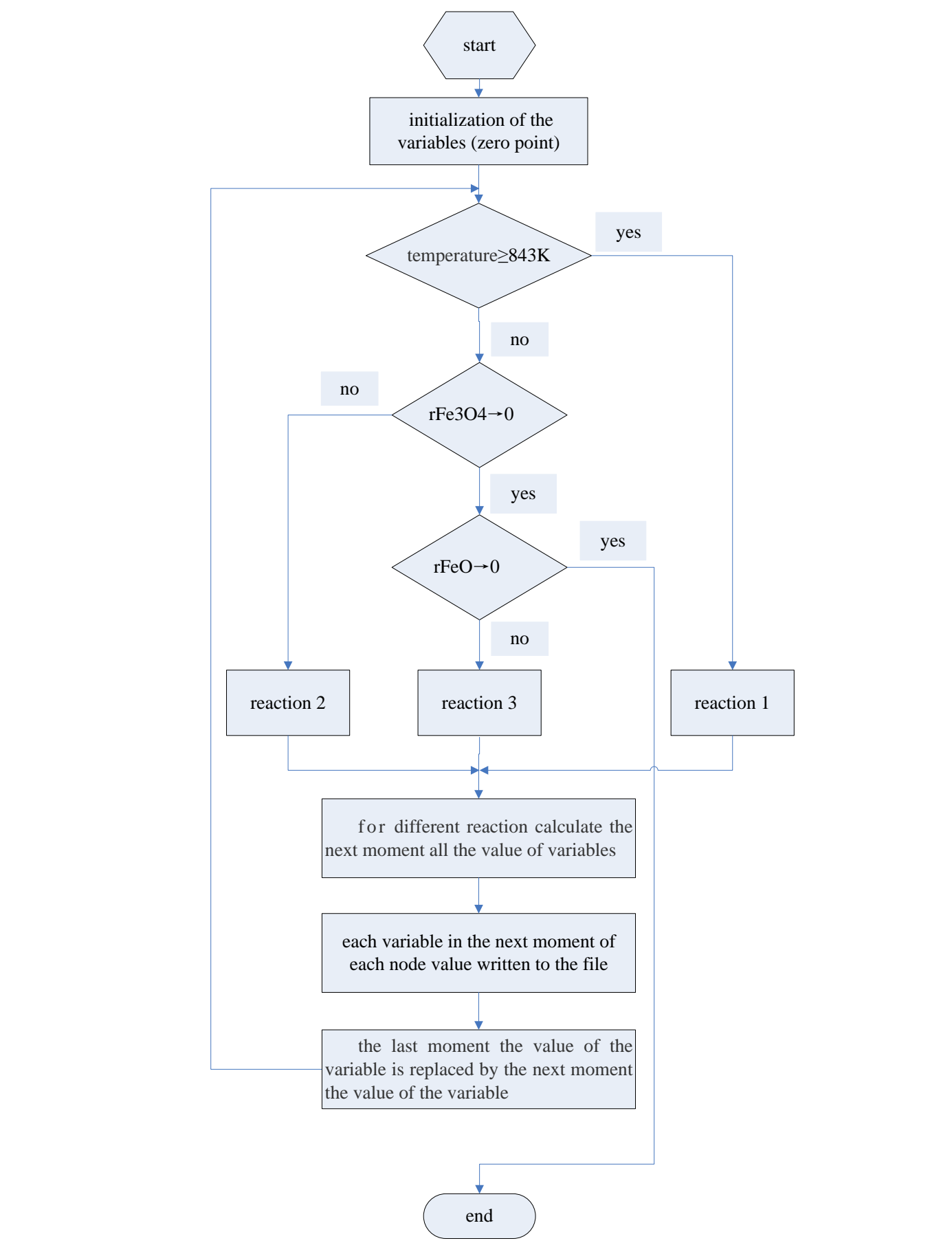

Figure 2. Computation procedure of the model

Calculated parameters are as follows: the thickness of iron ore is $30 \mathrm{~mm}$, and its particle sizes, density, heat capacity, thermal conductivity coefficient parameters are shown previously; reducing agent is $\mathrm{CO}$, not involved in $\mathrm{H}_{2}$.

Initial conditions: the layer temperature is $300 \mathrm{~K}$, gas is $100 \% \mathrm{CO}$. 
Boundary conditions:

(1) hot end: temperature is $1273 \mathrm{~K}$; reducing agent is $100 \% \mathrm{CO}$;

(2) cold end: thermal boundary is adiabatic boundary, one-way, no effect on gas export boundaries.

In the computation process, the spatial length of stride is $0.001 \mathrm{~m}$, the time length of stride is $0.001 \mathrm{~s}$, and the total overall reaction will not be finished until the computation carries on for $55 \mathrm{~s}$. The results are shown from Figure 3 to Figure 7.

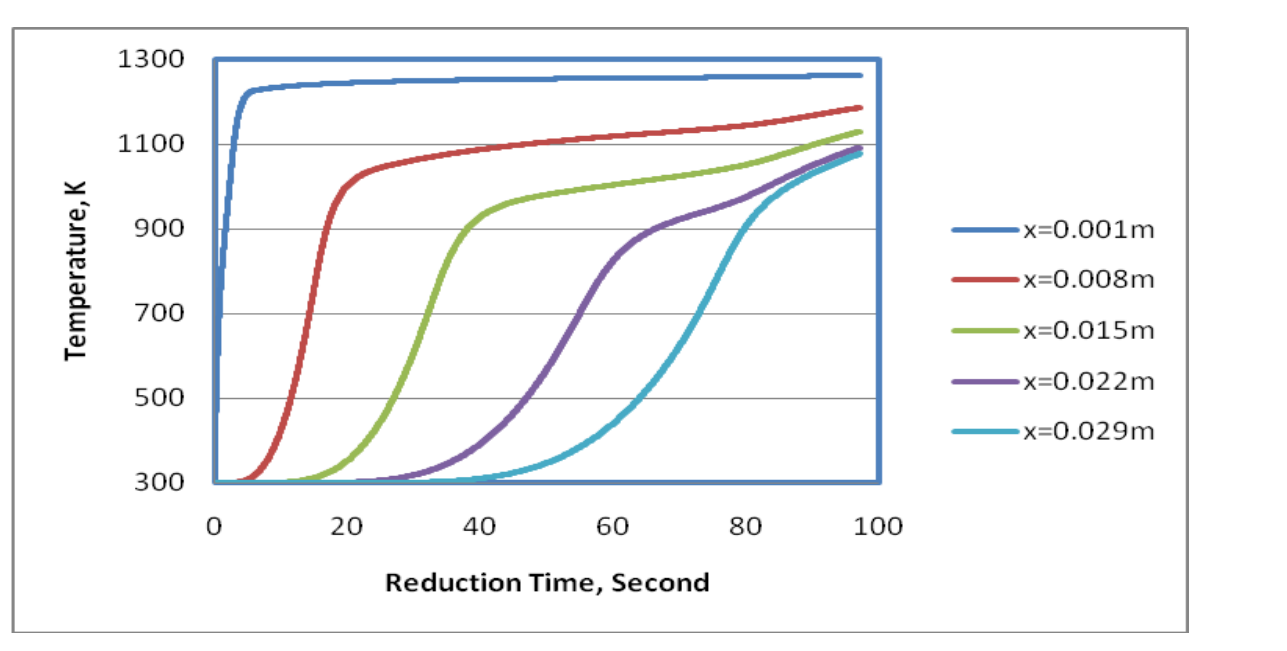

Figure 3. Relations between layer temperature and time

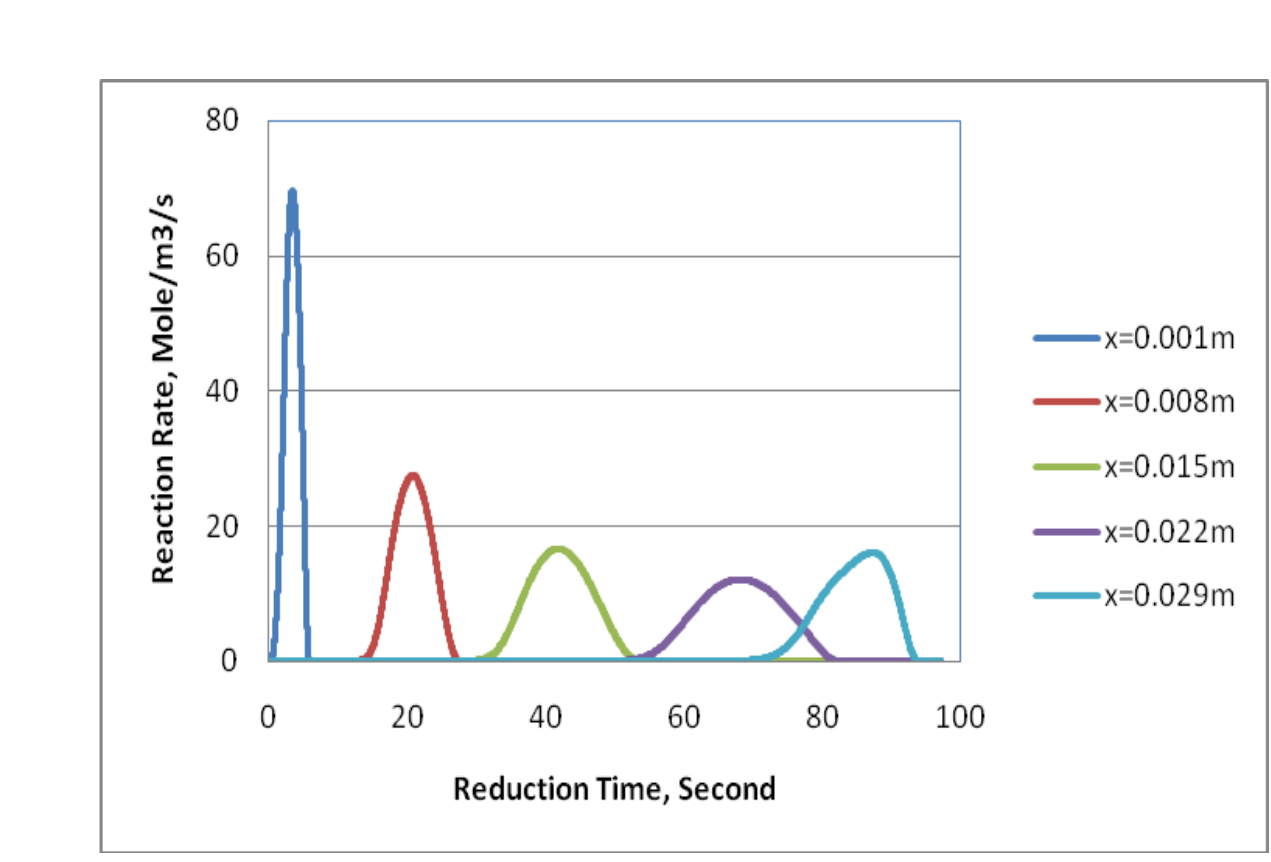

Figure 4. The curve of reduction rates changing with reduction time 


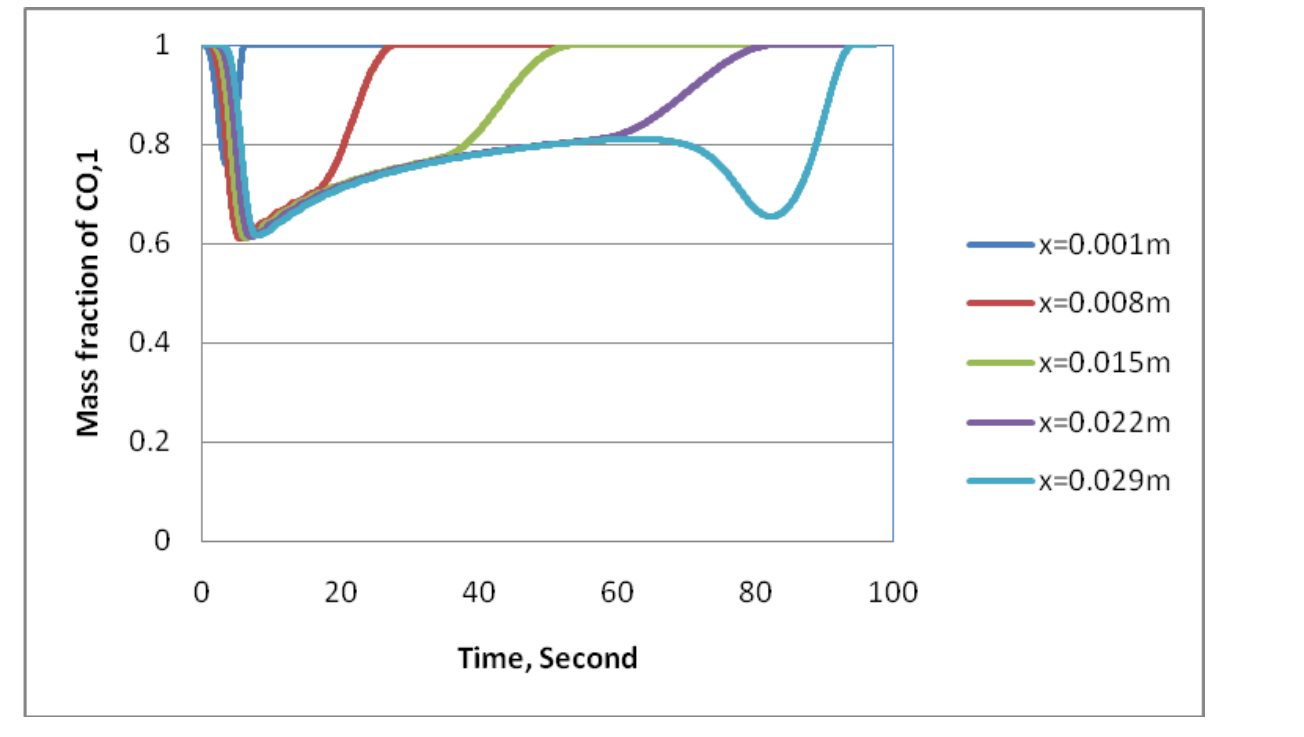

Figure 5. The curve of $\mathrm{CO}$ Componentvariation changing with reduction time

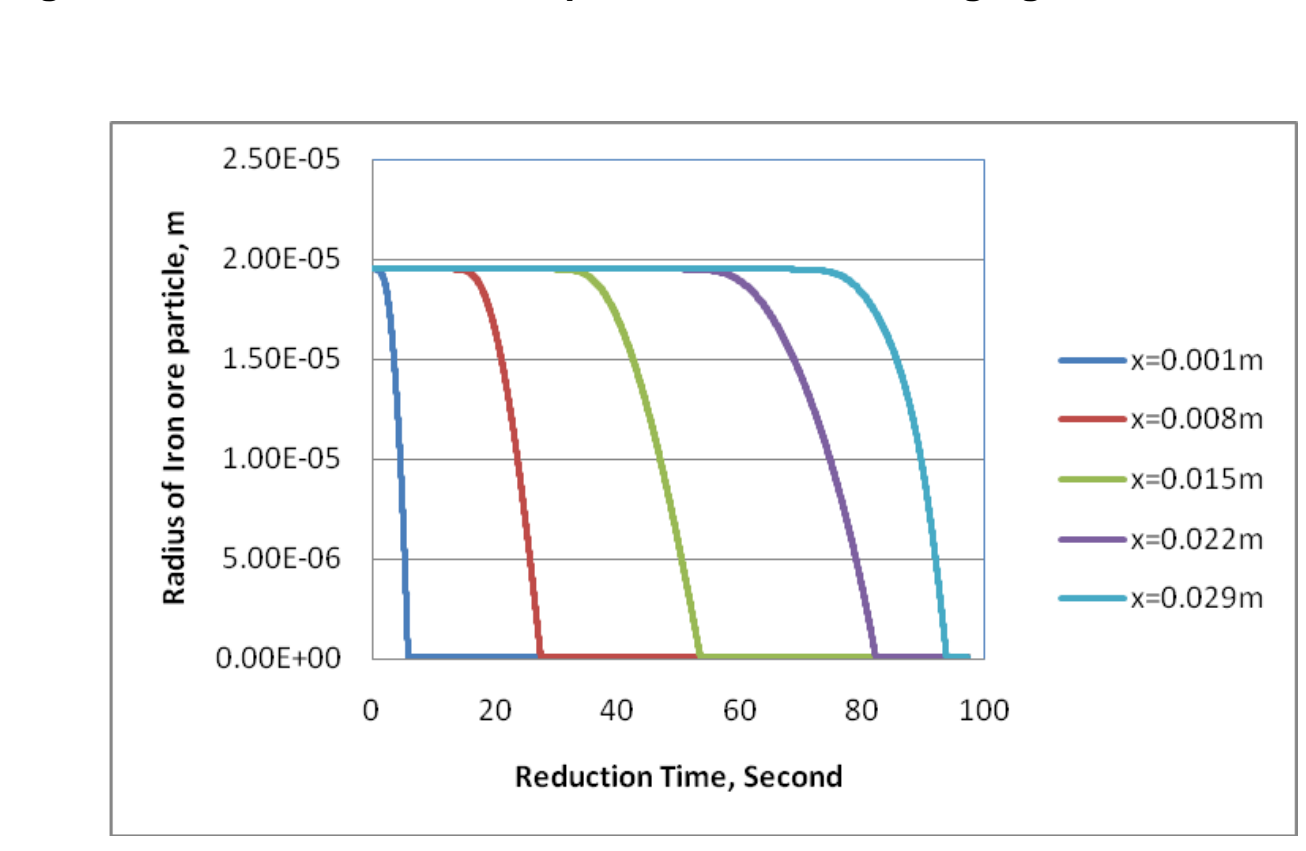

Figure 6. Size Changes of Mineral powder particles with reaction time 


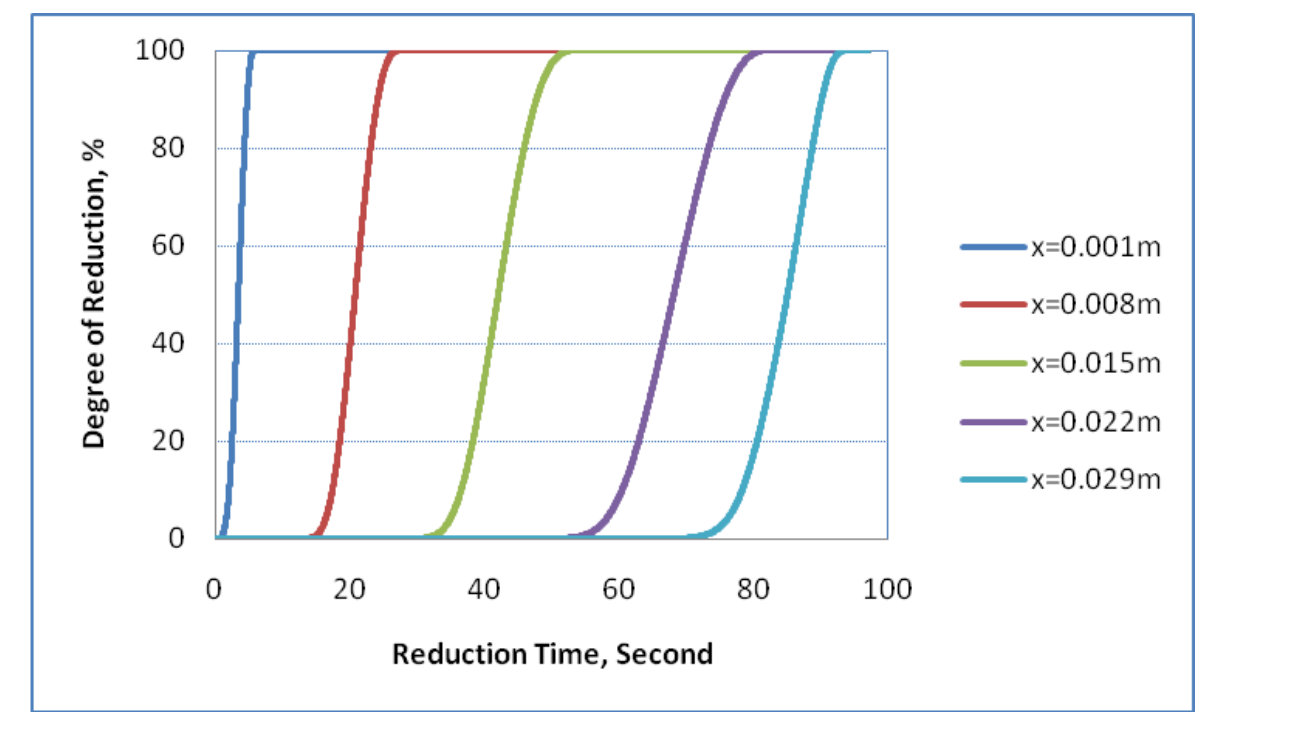

Figure 7. The curve of reduction degree changing with reduction time

\section{Conclusion}

According to the experimental research illustrated from Figure 3 to Figure 7, this research focuses on the selective feduction process of fine iron in reaction vessel, taking into consideration of following equations, such as heat transfer equation of porous medium, the rate equation of earbon gasified reaction, the equation of continuity, and the momentum equation andso on, and it establishes a micro dynamics mathematical model of the selective reduction process of fine iron and carbon mixture under the non-uniform temperature and non-equal-pressure conditions. Meanwhile, its results are reasonable, which confitms that the model can be applied to the simulation of reduction process of fine irongre

\section{Acknowledgements}

This research was supported by Natural Science Foundation of General Projects of Inner Mongolia Autonomous Region, China (2013MS0915) and Innovation Foundation Projects of Inner Mongolia University of Science \& Technology (2012NCL049).

\section{Nomenclature}

$$
\begin{aligned}
& \rho_{m}: \text { density of mixture }\left(\mathrm{kg} \cdot \mathrm{m}^{-3}\right) \\
& \rho_{g}: \text { density of gas }\left(\mathrm{kg} \cdot \mathrm{m}^{-3}\right) \\
& C_{p m}: \text { specific heat of solid mixtures }\left(\mathrm{J} \cdot \mathrm{kg}^{-1} \cdot \mathrm{K}^{-1}\right) \\
& C_{p g}: \text { specific heat of gases }\left(\mathrm{J} \cdot \mathrm{kg}^{-1} \cdot \mathrm{K}^{-1}\right) \\
& V_{0}: \text { superficial gas velocity }\left(\mathrm{m} \cdot \mathrm{s}^{-1}\right)
\end{aligned}
$$


$V_{a}:$ average velocity of gas fiow in the interstices of the packed bed $\left(m \cdot s^{-1}\right)$

$K_{\text {effm }}$ : effective thermal conductivity of the mixture $\left(W \cdot m^{-1} \cdot K^{-1}\right)$

$\dot{R}_{i}:$ rate of chemical reaction i $\left(\mathrm{mol} \cdot \mathrm{m}^{-3} \cdot \mathrm{s}^{-1}\right)$

$\Delta H_{i}$ : heat of chemical reaction i $\left(J \cdot \mathrm{mol}^{-1}\right)$

$S_{f}:$ shape factor of particles

$n_{i}$ : number of particles of solid reactant for reaction $\mathrm{i}$ in the mixture $\left.(m)^{3}\right)$

$\bar{r}_{i}$ : average radius of particle for reaction i $(m)$

$k_{i}:$ rate constant for reaction i $\left(m \cdot s^{-1}\right)$

$k_{i 0}$ : Pre-exponential constant in rate constant for reaction i

$K_{E i}$ : equilibrium constant for reaction 1

$\Delta E_{i}^{*}$ : 'activation energy' of reaction i $\left(J \cdot\right.$ mot $\left.^{-1}\right)$

$R$ : gas constant $\left(8.314 \mathrm{~J} \cdot \mathrm{mol}^{-1} \cdot \mathrm{K}^{\mathrm{A}}\right)$

$C_{C O}$ : concentration of $\mathrm{CO}\left(m \varnothing l \cdot \mathrm{m}^{-3}\right)$

$\mathrm{C}_{\mathrm{CO}_{2}}$ : concentyation of $\mathrm{EO} 2\left(\mathrm{~mol} \cdot \mathrm{m}^{-3}\right)$

$C_{H_{2}}$ : concentrationor $\mathrm{H} 2\left(\mathrm{~mol} \cdot \mathrm{m}^{-3}\right)$

$\mathrm{C}_{\mathrm{H}_{2} \mathrm{O}}$ : concentration of $\mathrm{H} 2 \mathrm{O}\left(\mathrm{mol} \cdot \mathrm{m}^{-3}\right)$

$W_{v i}$ : un-eyolved portion of volatile component i $(\mathrm{kg} / \mathrm{kg}-\mathrm{coal})$

1. binary diffusivity in the gaseous phase $(\mathrm{m} 2 / \mathrm{s})$

$y_{j}$ : mole fraction of species $\mathrm{j}$ in the gaseous phase $(\%)$

$\phi$ : void fraction of the packed bed

$\rho_{j}:$ density of gaseous species $\mathrm{j}\left(\mathrm{kg} \cdot \mathrm{m}^{-3}\right)$

$\dot{N}_{i j}$ : net rate of gaseous species j consumed/generated by chemical reaction i ( $\left.\mathrm{mol} \cdot \mathrm{s}^{-1} \cdot \mathrm{m}^{-3}\right)$ 


$$
\begin{aligned}
& M_{j}: \text { molecular weight of the gaseous species j }\left(\mathrm{kg} \cdot \mathrm{mol}^{-1}\right) \\
& \tau: \text { tortuosity factor } \\
& \mu: \text { viscosity of fluid }\left(\mathrm{kg} \cdot \mathrm{m}^{-1} \cdot \mathrm{s}^{-1}\right)
\end{aligned}
$$

\section{References}

[1] S. Sun, "A Study of Kinetics and Mechanisms of Iron Ore Reduction in Ore/Coal Composites", (1997).

[2] H. Dianbing, Y. Xuemin and Y. Tianjun, "Reduction Process of Carbon-bearing Pellets and its Model", Journal of Metals, vol. 32, no. 6, (1996), pp. 629-636.

[3] E. Donskoi, D. L. S. Mcelwain and L. J. Wibberley, "Estimation and Modeling of Parameters for Direct Reduction in Iron Ore/Coal Composites: Part2. Kinetic Parameters", Metallurgical and Matérials Transaction B, vol. 34B, (2003), pp. 255-266.

[4] T. Huiqing, G. Xingmin, Z. Shengbi and G. Zhancheng, "The Model of Reduction kinetics of carbon-bearing pellets under $\mathrm{CO} / \mathrm{CO} 2$ climate and its application”, Journal of joo and steel résearch, vol. 12, no. 6, (2000), pp. 1-6.

[5] Q. Wang, Z. Yang, J. Tian, et al., "Reduction Kinetics of Iron Ore-Coal Pellet During Fast Heating", Ironmaking and Steelmaking, vol. 25, no. 6, (1998), pp. 443-447.

[6] E. Donskoi and D. L. S. McElwain, Applied Mathemafics Letters, (1999), pp. 27-34.

[7] E. Donskoi and D. L. S. McElwain, Metallurgical and Materials Transactions, vol. 34B, (2003), pp. 93-102.

[8] X. Hui, Z. Zongshu and Y. Aibing, "Numerical simulation of Midrex reduction shaft furnace reaction", iron and steel, (2008).

[9] X. Hui and Z. Zongshu, "Research on modeling of Direct Iron Reduction Process in Shaft Furnace", Iron and Steel in the World, (2009).

[10] M. D. Zhimin, "Research on Direct reduction of iron ore tailings", China Metallurgical, (2010).

[11] B. Wilkinson and M. Allen, "ParalleI Pprogran Designing", (Translated by Lu Xin) Beijing: China machine press, vol. 5, (2005), pp. 189 193.

[12] OpenMP, http://www.openmp.org/drupal/.

[13] C. Guoliang, A. Hong and C. Ling, "Parallel Athmetic Practice", Beijing: higher education press, (2004).

[14] K. Liu and Kouzheng, "Applications of OpenMP in Parallel Computing", Microcomputers and Application, vol. 12, (2003), $\mathrm{pp}, 12-14$.

[15] M. yan, "Mulficore: Expanding history along the Trajectory", Programmer, vol. 9, (2006), pp. 2 2.

[16] Y. Li, "Realization of MPI paallel computing of casting filling simulation", Master degree thesis, Chongqing: Chongqing University, (2098)

[17] S. Hanqi, "Study of parallel computing in computational fluid dynamics", master degree thesis, Liaoning: Dalian University of technology, (2005).

[18] L. Shilai and X. Xuejun, "OpenMP applications in parallel computation of hydrodynamic mathematical model”, Marine Engineering, vol. 8, (2010).

[19] C. Bangqian, "Research and Developing of Parallel Computing in Casting Numerical Simulation", master degree thesis, Wuhan: Hua Zhong University of Science and Technology, (2007).

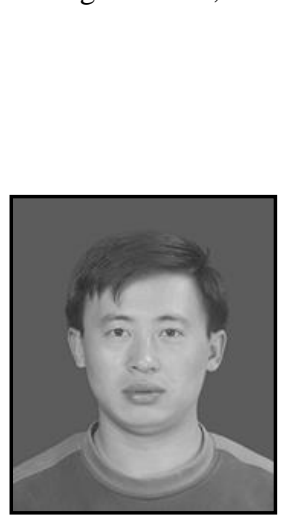

\section{Author}

Lan Xiaowen received the BS in computer science from Inner Mongolia University, China, in 2001, and received the MS in computer science from Chengdu University of Technology, China, in 2008. Currently, his research interests include embedded system and scheduling techniques and parallel algorithms for clusters, and also multi-core processors and numerical simulation. 